\title{
New device for real-time bioluminescence imaging in moving rodents
}

Emilie Roncali

Laboratoire d'Imagerie Moléculaire Expérimentale SHFJ, I2BM, CEA

Inserm U 8034 place Leclerc

91400 Orsay, France and

Biospace Lab

10 rue Mercoeur

75011 Paris, France

\section{Mickael Savinaud}

Biospace Lab

10 rue Mercoeur

75011 Paris, France

and

Ecole Centrale Paris

Laboratoire MAS

Grande Voie des Vignes

92290 Chatenay Malabry Cedex, France

\section{Olivier Levrey}

Biospace Lab

10 rue Mercoeur

75011 Paris, France

\section{Kelly L. Rogers*}

Laboratoire d'Imagerie Moléculaire Expérimentale SHFJ, I2BM, CEA

Inserm U 8034 place Leclerc

91400 Orsay, France

\section{Serge Maitrejean}

Biospace Lab

10 rue Mercoeur

75011 Paris, France

\section{Bertrand Tavitian}

Laboratoire d'Imagerie Moléculaire Expérimentale SHFJ, I2BM, CEA

Inserm U 8034 Place Leclerc

91400 Orsay, France

E-mail: bertrand.tavitian@cea.fr

\begin{abstract}
Bioluminescence imaging (BLI) allows detection of biological functions in genetically modified cells, bacteria, or animals expressing a luciferase (i.e., firefly, Renilla, or aequorin). Given the high sensitivity and minimal toxicity of BLI, in vivo studies on molecular events can be performed noninvasively in living rodents. To date, detection of bioluminescence in living animals has required long exposure times that are incompatible with studies on dynamic signaling pathways or nonanaesthetised freely moving animals. Here we develop an imaging system that allows: 1 . bioluminescence to be recorded at a rate of 25 images/s using a third generation intensified charge-coupled device (CCD) camera running in a photon counting mode, and 2. coregistration of a video image from a second CCD camera under infrared lighting. The sensitivity of this instrument permits studies with subsecond temporal resolution in nonanaesthetized and unrestrained mice expressing firefly luciferase and imaging of calcium signaling in transgenic mice expressing green fluorescent protein (GFP) aequorin. This imaging system enables studies on signal transduction, tumor growth, gene expression, or infectious processes in nonanaesthetized and freely moving animals. ๑ 2008 Society of Photo-Optical Instrumentation Engineers. [DOI: 10.1117/1.2976426]
\end{abstract}

Keywords: luminescence; photon counting camera; whole body imaging; high temporal resolution; moving animals; unanesthetized; video tracking; registration.

Paper 08002R received Jan. 3, 2008; revised manuscript received May 19, 2008; accepted for publication Jun. 4, 2008; published online Sep. 25, 2008.

\section{Introduction}

Molecular imaging is a noninvasive method for studying the chemistry of life in whole living organisms such as rodents. This rapidly expanding field is driven by innovative developments, constantly offering new tools for imaging the molecular basis of biological processes.

*Present address: Institut Pasteur, Plate-forme d'Imagerie Dynamique, 25 Rue du Docteur Roux 75724, Paris Cedex 15, France.

Address all correspondence to Bertrand Tavitian, Laboratoire d'Imagerie Moléculaire Expérimentale, SHFJ, I2BM, CEA, Inserm U 8034 Place Leclerc, 91400 Orsay, France; Tel: +33 16986 7779; Fax: +33 16986 7786; E-mail: bertrand.tavitian@cea.fr.
To date, molecular imaging in whole animals has been restricted to relatively lengthy time scales (generally seconds to minutes). Molecular imaging has therefore been limited to anaesthetized or restrained animals. Physiological experiments do not take motion into account, despite its importance in studies on animal cognition or neuromuscular diseases. ${ }^{1}$ Moreover, rapid and transient phenomena such as signal transduction processes could not be kinetically investigated with accuracy by in vivo noninvasive techniques. It has been also reported that anesthesia can induce cytotoxicity in PC12

1083-3668/2008/13(5)/054035/10/\$25.00 @ 2008 SPIE 
cells ${ }^{2}$ and blood flow variations, ${ }^{3}$ and has influences on the uptake of nuclear tracers. ${ }^{4}$ Performing molecular imaging studies without the use of anaesthetics and in freely moving animals would limit the interference with signalization processes. Consequently, behavioral studies and brain activity monitoring on awake rodents have been undertaken using imaging modalities such as functional magnetic resonance imaging (fMRI), but most experiments were realized on restrained animals. ${ }^{5,6}$ Single photon emission computed tomography (SPECT) has also been performed on awake animals that were placed into a cylinder. ${ }^{7}$ Standard fluorescence and twophoton microscopy experiments have been realized in freely moving animals, ${ }^{8}$ but they are limited to a specific part of the body. Hence, despite the fact that they offer detailed information with great spatial resolution, they do not allow whole body imaging.

Among the different modalities of molecular imaging, ${ }^{9}$ luminescence emission-based techniques appear the most attractive for the development of high temporal resolution imaging. Notably, the use of endogenous markers such as reporter proteins $^{10-13}$ allows observation of phenomena in a noninvasive way, which is critical for gathering data in physiological conditions and for performing longitudinal studies. Moreover, these approaches can be performed at high temporal resolution, because photons are produced at a high frequency in the visible range.

Bioluminescence has a great advantage over fluorescence, because no exogenous excitation light is required. No background is produced, leading to a good signal-to-noise ratio (SNR) and enabling biological events having fast kinetics to be detected by adapted instruments. ${ }^{14}$ However, the absence of background signal in bioluminescence makes the localization of the contour of the animal impossible. Therefore, imaging on moving animals requires an additional tracking system to follow animal motion.

Because of their sensitivity, cooled charge-coupled device (CCD) cameras are largely used to detect the very small amount of light produced by bioluminescence reactions or by fluorescence probes. However, given that signals encountered in bioluminescence studies are by far lower than fluorescence emissions, these systems require an exposure time of several seconds to overcome the readout noise. ${ }^{15}$ This is critical for dynamic signaling pathway studies or behavior mechanism investigations. A similar problem would appear if experiments were performed in nonanaesthetized or unrestrained animals, as the animal movement during these few seconds would blur the luminescent signal. To achieve such experiments, imaging with high temporal resolution is necessary. Photon-countingbased systems, such as intensified CCD cameras, are particularly relevant because of their great sensitivity and their low noise at short exposure times. ${ }^{16}$ They offer temporal resolutions down to $20 \mathrm{~ms}$ and are therefore compatible with transitory biological process observations over the visible spectrum, as their quantum efficiency is almost constant from 450 to $850 \mathrm{~nm}$.

We report the development and validation of a photoncounting-based system combined with a video monitoring function. The biological signal of interest, recorded by a third generation cooled intensified CCD camera, is spatially and temporally registered with the tracking video. With infrared lighting the motion of the animal is imaged without disrupting the acquisition of the bioluminescence emitted from its body. A description of the apparatus is presented, together with the experimental validation of its ability to associate high temporal resolution and sensitive imaging. This technique was applied to in vivo imaging of a freely moving mouse bearing a luciferase-expressing tumor and visualization of calcium signaling in mouse during stimulated muscle contractions.

\section{Technical Description of the Instrument}

Whole body optical imaging of small animals was undertaken using an intensified CCD camera (ICCD) operating in a photon counting mode (Photon Imager ${ }^{\mathrm{TM}}$, Biospace Lab, France). The camera is made up of an f/1.4 lens and a third generation light intensifier tube (GaAs photocathode) set at a high gain level $\left(10^{6}\right)$. The intensifier is tapered to a half-inch CCD chip, which gives a spatial resolution of $200 \mu \mathrm{m}$ at the object plane. A light spot is produced by the light intensifier each time a photon hits the photocathode, and this light spot is detected by the CCD so that each incident photon can be recorded. This intensified camera (ICCD) is mounted on top of a light-tight chamber $(30 \mathrm{~cm}$ diam $)$ to record optical signals at a video rate of $25 \mathrm{~Hz}$. Images are acquired as dynamic sequences with a 40-ms temporal resolution and depicted in real time on a screen display. The user can sum any number of consecutive frames by postprocessing to improve the detection of variable signal kinetics.

The read-out noise of the $\mathrm{CCD}$ can be eliminated by thresholding, thanks to the amplification process taking place in the intensifier tube (the gain is about $10^{6}$ ) before the readout operation. Hence, the noise is only limited by the thermal noise of the GaAs photocathode, which is cooled down to $-25^{\circ} \mathrm{C}$. At this temperature, the dark count is in the range of 100 detected photons per second on the whole area for an 18-mm tube, which means that short signals can be detected with a much higher signal-to-noise ratio than what can be achieved with other cooled CCDs.

Motion should also be monitored in experiments with awake and unrestrained animals. We designed a system consisting of two cameras, one for recording the signal of interest and a second for video tracking the animal. The two signals from the two cameras are simultaneously recorded and electronically synchronized.

For video tracking of the animal during motion, the field of view is illuminated by an infrared light [Fig. 1(a)], provided by a $50-\mathrm{mW}$ laser diode emitting at $785 \mathrm{~nm}$ (Power Technology, Incorporated Alexander, Arkansas). The infrared (IR) illumination avoids spectral overlap with the emission spectrum of most common bioluminescent probes [Fig. 1(b)]. To generate the tracking video, the infrared illumination reflected by the animal is recorded at $25 \mathrm{~Hz}$ by a half-inch CCD camera (JAI A-S, Copenhagen, Denmark) coupled with an 8-mm focal length objective (Edmund Optics Limited, York, United Kingdom). This infrared camera is placed at 90 deg with respect to the ICCD axis in the chamber, and has a sensitivity of $50 \mathrm{mLux}$. The field of view of $20 \times 15 \mathrm{~cm}$ is slightly larger than the ICCD field of view (FOV) and sufficient for the mouse to move. The spatial resolution of the video image is also around $200 \mu \mathrm{m}$.

The optical signal coming from the animal is a mixture of the reflected lighting and the bioluminescence signal. This 
(a)

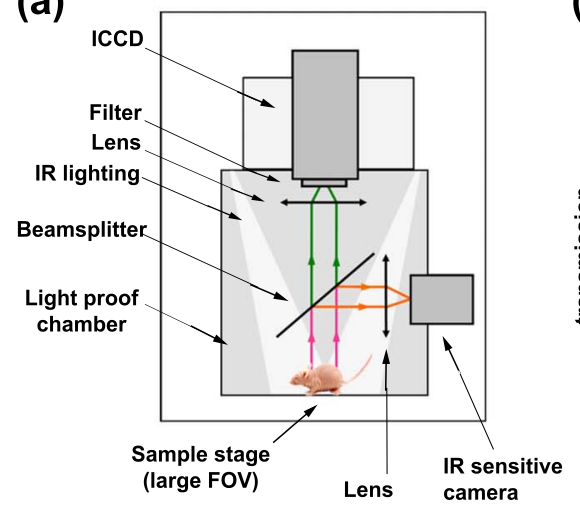

(b)

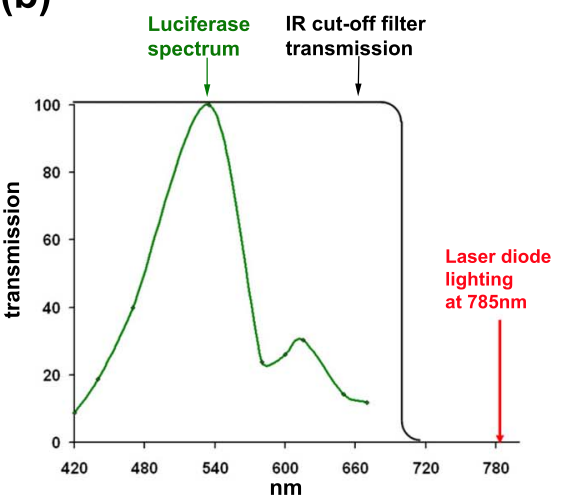

(c)

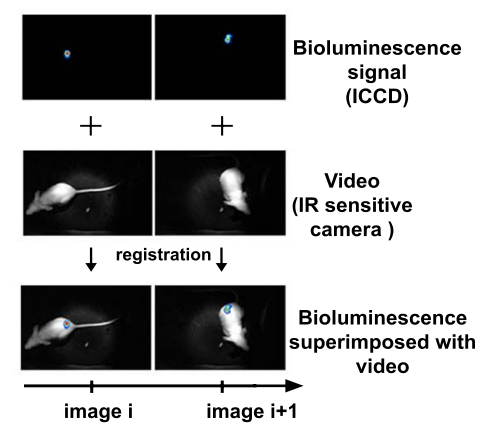

Fig. 1 Optical setup and schematic description of the acquisition. (a) Optical setup inside the light proof chamber. Illumination was performed with an infrared (IR) lighting partially reflected by the beamsplitter and recorded by an IR sensitive CCD camera. Bioluminescence signals were recorded with an ICCD. (b) Schematic transmission spectra of IR cut-off filter, luciferase emission, and IR illumination. (c) Schematic representation of acquisition and overlay process.

light is split into two orthogonal shafts and recorded on separate optical pathways. The splitting is operated by a $45 \times 60$-mm beamsplitter (Omega Optical, Incorporated, Vermont) placed at an angle of $45 \mathrm{deg}$ with respect to the vertical axis. The beamsplitter transmits visible light (average transmission of $90 \%$ over $[400 ; 700 \mathrm{~nm}]$ ) while it reflects infrared light over $[700 ; 1100 \mathrm{~nm}]$ due to its dichroic coating. The optical density (OD) of the beamsplitter over $[700 ; 1100 \mathrm{~nm}]$ is 2. An IR cut-off filter with a steep edge at $700 \mathrm{~nm}$ and a theoretical optical density superior to 6 (Barr Associates, Incorporated, Massachusetts) is placed in front of the camera to eliminate the residual illumination. Based on the minimum lighting required for the video tracking and the ICCD sensitivity, we calculated that the filtering should have a minimum OD of 10 over the blocking range, so a short-pass filter with a similar spectrum is added to increase the total blocking. Spectral data provided by the manufacturers for each filter showed an average transmission of $90 \%$ over the transmission range $(400$ to $700 \mathrm{~nm})$. This leads to a global transmission of $70 \%$ $\left(0.9^{3}\right)$ for the combined optical components.

To compensate for the difference of FOVs, we conduct a global registration on the two images from the two cameras. This registration is an affined transformation performed in real time during the acquisition, allowing the superimposed image (bioluminescence plus video image) to be properly displayed [Fig. 1(c)].

\section{Experimental Validation of the System}

For simplicity, basic bioluminescence recording is referred to in this work as "standard BLI mode," whereas bioluminescence imaging together with the video monitoring function (with filters and IR illumination) is the "BLI video mode." The instrumental setup was validated in vitro and then in vivo by comparing these two modes. If not specified, acquisitions were performed for $3 \mathrm{~min}$ with the largest aperture number (1.4).

All signal measurements were done using region of interest (ROI) on raw data, and smoothing was applied for the purpose of display only.
SNR was defined by measurement of the radiance (photons $/ \mathrm{s} / \mathrm{cm}^{2} / \mathrm{sr}$ ) in same size ROIs enclosing areas containing signal and areas of the image without signal (background). Background was measured on all frames within a sequence for calculation of the standard deviation $(\sigma)$, and the SNR was calculated according to the following equation:

$$
\mathrm{SNR}=\operatorname{signal} / \sigma
$$

\subsection{Evaluation of Transmission, Linearity, and Sensitivity on Static Sources}

The following studies were undertaken to optimize the optical setup.

The background was measured on a whole image in both acquisition modes without any light source. In standard BLI mode, the noise radiance was found to be $680 \mathrm{ph} / \mathrm{s} / \mathrm{cm}^{2} / \mathrm{sr}$, whereas it was about $980 \mathrm{ph} / \mathrm{s} / \mathrm{cm}^{2} / \mathrm{sr}$ in BLI video mode (images not shown).

Transmissions were evaluated with two low intensity diodes, at two different wavelengths (green at $525 \mathrm{~nm}$; red at $625 \mathrm{~nm})$. Signals were first measured in standard BLI mode [Fig. 2(a)], without additional optical elements or lighting, then second with the beamsplitter only, then third with the filters only, and finally with all elements together. Images were also acquired using the same protocol but adding the illumination (BLI video mode). All transmissions were measured for the complete duration acquisition $(3 \mathrm{~min})$. Consistent with spectra provided by the manufacturers, $70 \%$ of the signal is transmitted at $625 \mathrm{~nm}$ and $69 \%$ at $525 \mathrm{~nm}$ [Fig. 2(b)].

Experiments were carried out on NIH-3T3 cells stably expressing the luc gene (Promega Corporation, Madison, Wisconsin), to compare the linearity of BLI video mode to the standard BLI mode. Cells were serially diluted in a blackbottom 96-well plate, and $20 \mu \mathrm{L}$ (for a final volume of $200 \mu \mathrm{L}$ ) of beetle luciferin (Promega Corporation, Madison, Wisconsin) at $1 \mathrm{mM}$ was added to trigger the reaction [Fig. 2(c)]. Reaction of the beetle luciferin with the expressed luciferase was immediate, and light emission reached a plateau 
(a)

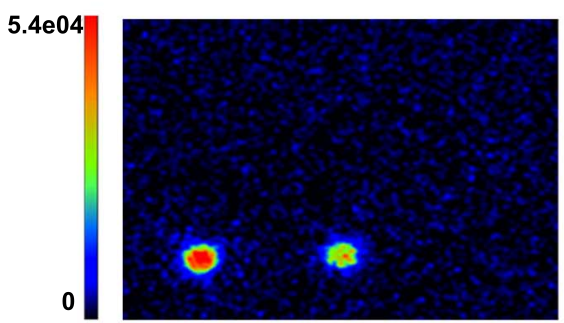

(c)

\section{(i) Standard BLI mode}

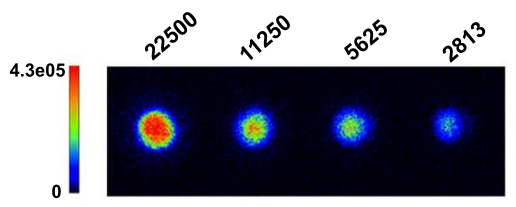

(ii) Real time acquistion mode

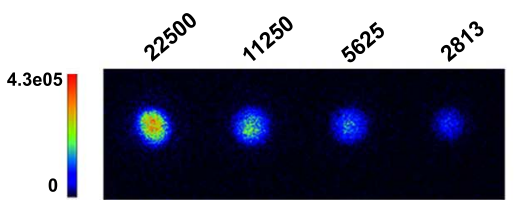

(b)

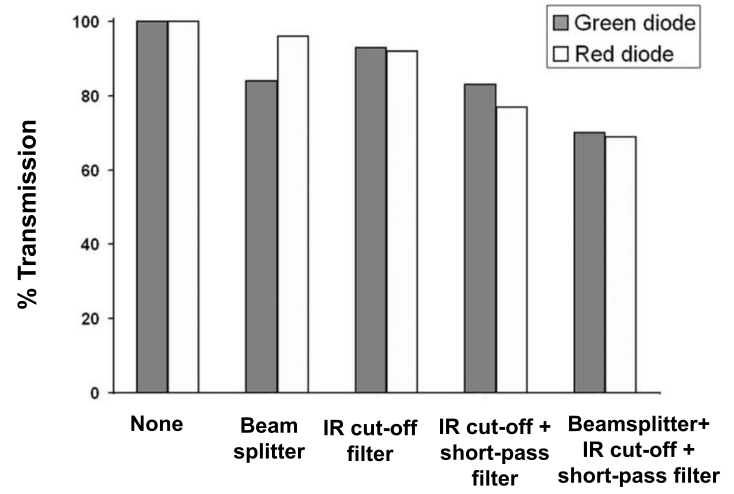

(d)

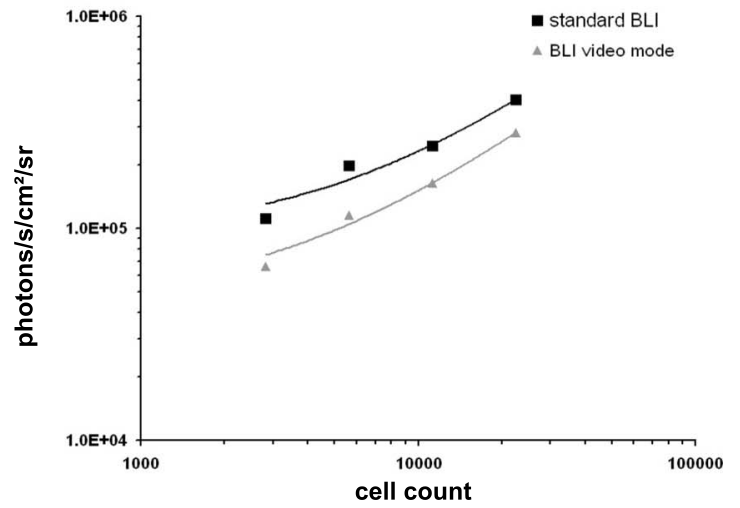

Fig. 2 In vitro evaluation of transmission and linearity. (a) 3-min images (0.6-mm smoothing; photon $/ \mathrm{s} / \mathrm{cm}^{2} / \mathrm{sr}$ ) of two diodes (green, red). (b) Transmissions on the complete sequence duration for different filtering combinations ( $70 \%$ with all the filtering elements). (c) Recordings on serial dilution of NIH-3T3 luc2 cells (3-min exposure time; photon $/ \mathrm{s} / \mathrm{cm}^{2} / \mathrm{sr}$ ) in (i) standard BLI mode and (ii) BLI video mode. (d) Log-log plot of the light radiance (photon $/ \mathrm{s} / \mathrm{cm}^{2} / \mathrm{sr}$ ) versus cell count for the standard BLI (solid line) and BLI video modes (dashed line) show good linearity $\left(R^{2}\right.$ $=0.97$ and $R^{2}=0.99$, respectively). (Color online only.)

within $30 \mathrm{~s}$. Subsequent studies were therefore undertaken in conditions where steady-state light emission was maintained. To control possible variations in light emission due to luciferase/luciferin reaction kinetics, the light radiance was measured in BLI standard mode, followed by BLI video mode, and then again in BLI standard mode. Plots of light radiance versus cell count in standard BLI and BLI video mode indicate that the relation between the number of cells and total light emission is linear in both acquisition modes, with a $30 \%$ reduction in the amount of light detected in the BLI video mode due to the use of filters [Fig. 2(d)].

Evaluations of transmissions and SNR were done on anaesthetized nude mice bearing two xenografts of luciferaseexpressing NIH-3T3 tumor cells.

All animal experiments were approved by the local animal ethics committee and conducted in accordance with current French and European legislation.

The mouse anaesthetized with isoflurane was injected (intraperitoneal injection) with luciferin $(300 \mu \mathrm{L}$ of $1.5 \mathrm{mg} / \mathrm{mL}$, Promega Corporation, Madison, Wisconsin), and then placed inside the chamber [Fig. 3(a)]. The kinetics of light emitted by these tumors was found to remain relatively constant throughout the whole acquisition [Fig. 3(b)]. SNRs were calculated for different combinations of optical elements and lighting [Fig. 3(c)]. In BLI video mode, the SNR for a 3-min acquisition was 1833 , representing as expected $68 \%$ of the standard BLI mode SNR. Finally, SNR values for exposure times between $40 \mathrm{~ms}$ and $1 \mathrm{~s}$ were between 24 and 120 [Fig. 3(d)]. In line with expectations, the relation between SNR and exposure time is a square root function $\left(R^{2}\right.$ $\approx 0.99$ ).

These results demonstrate that the signal acquisition with this system could be done properly with static sources, and that light measurements could be easily performed. In further studies, the system was validated for moving light sources. We assume that the light intensity measured on a ROI adapted to the source location is comparable to the signal intensity measured on the complete field of view. To validate that the motion does not induce a loss of signal, these two intensities were compared after the background was subtracted. In addition, measurement methods adapted to a moving area of light emission were applied and evaluated.

\subsection{Signal Recording and Measurement Methods Evaluation on Moving Mice}

In anaesthetized mice, the SNR reached 24 for an exposure time of $40 \mathrm{~ms}$, suggesting that the system could be suitable for imaging moving animals. On the same mouse, the anesthetic gas input was therefore switched off and the mouse was 
(a)

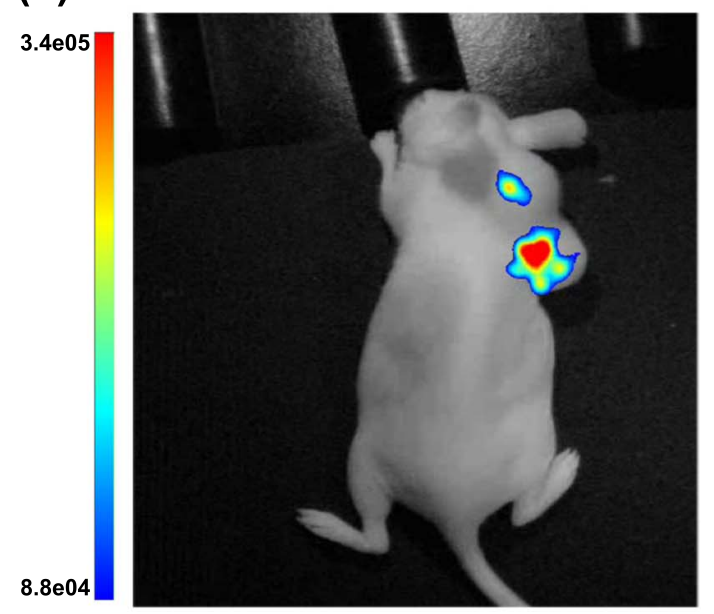

(c)

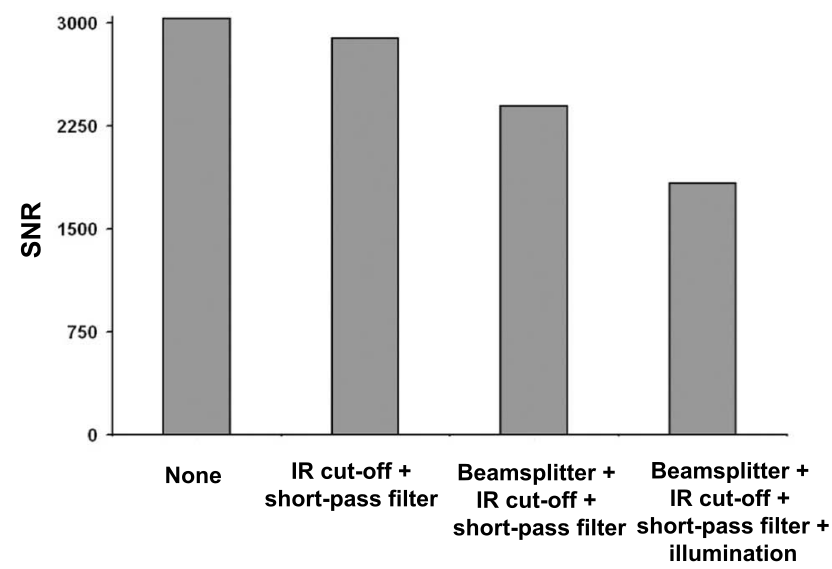

(b)

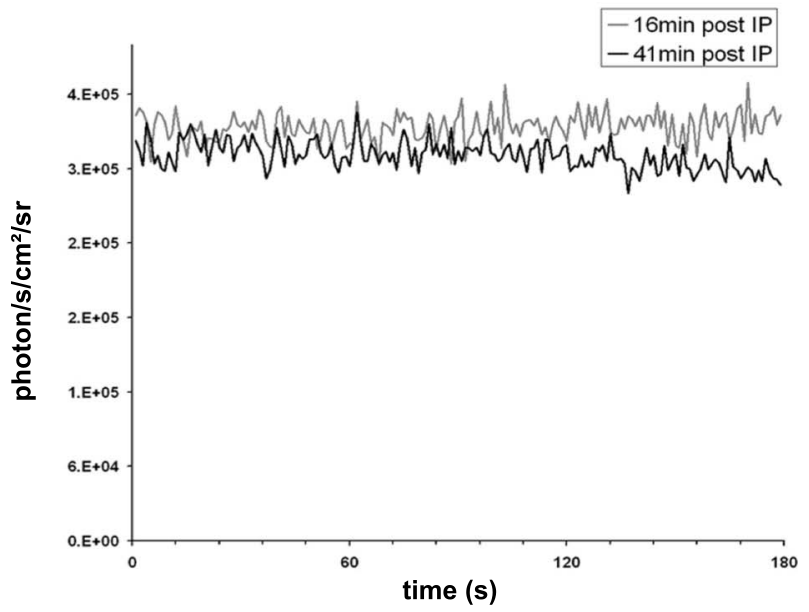

(d)

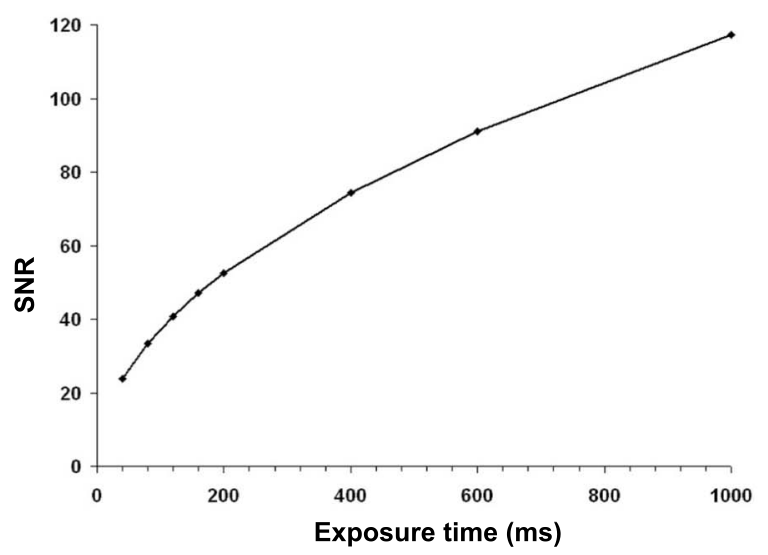

Fig. 3 SNR and transmission measurements on anaesthetized nude mouse. (a) 200-ms images of bioluminescence emission (5-mm smoothing; photon $/ \mathrm{s} / \mathrm{cm}^{2} / \mathrm{sr}$ ) superimposed with the video image of the animal. (b) Kinetics acquired 16 and 41 min after IP injection (gray and black curve, respectively). (c) Comparison of SNR in different modes for the whole acquisition duration. SNR in BLI mode is $68 \%$ of the standard BLI mode value. (d) SNR measured on a ROI containing the two tumors versus exposure time. Values vary between 24 and 120 for exposure times from $40 \mathrm{~ms}$ to $1 \mathrm{~s}$.

left to wake up gently in the black chamber. Movements were monitored during several acquisitions, and one sequence is shown on Video 1. Two ROIs situated on the mouse trajectory at different time periods were drawn manually [Fig. 4(a)]. Corresponding time courses of light intensity were compared to the time course of the total signal (after background subtraction) measured on the complete FOV [Fig. 4(b)]. As expected with the manual ROIs that were used, signal intensities in these ROIs were slightly less than the light intensity obtained for the complete FOV. SNR was calculated over a specific time period, using an ROI containing the signal in this time period [ROI drawn in red in Fig. 4(a)] and an identical ROI located in the background. This procedure was replicated for seven exposure times between $40 \mathrm{~ms}$ and $1 \mathrm{~s}$. Similar measurements were performed on five different sequences, and the mean SNR versus exposure time is shown in Fig. 4(c). The bars represent the minimum and maximum values obtained for all the sequences at each exposure time, and establish that a variation around $30 \%$ could be observed through

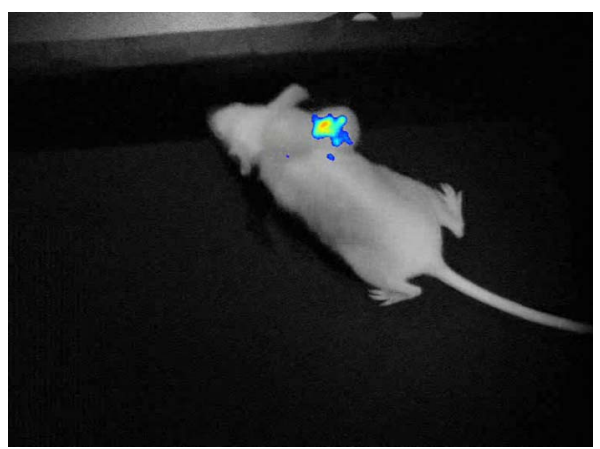

Video 1 A freely moving nude mouse bearing subcutaneous tumors expressing luciferase. Movements and bioluminescence signal have been recorded simultaneously. Video 1 was generated after the experiment with an exposure time of $200 \mathrm{~ms}$ and 5-mm smoothing (QuickTime, 2.33 MB)

[URL: http://dx.doi.org/10.1117/1.2976426.1]. 
(a)

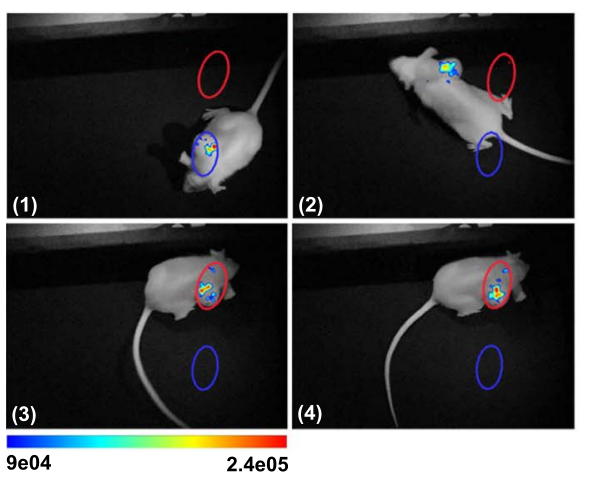

(b)

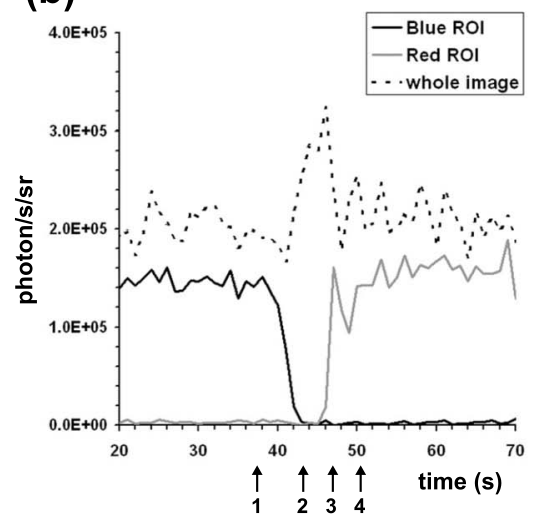

(c)

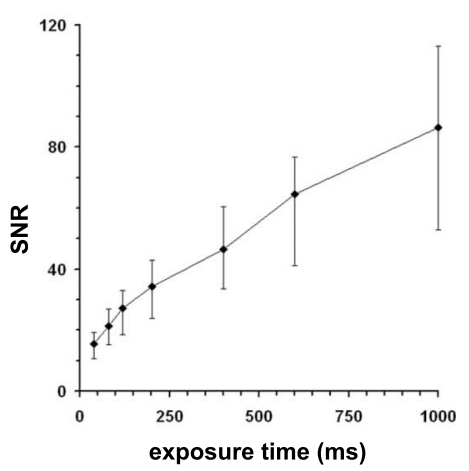

(d)

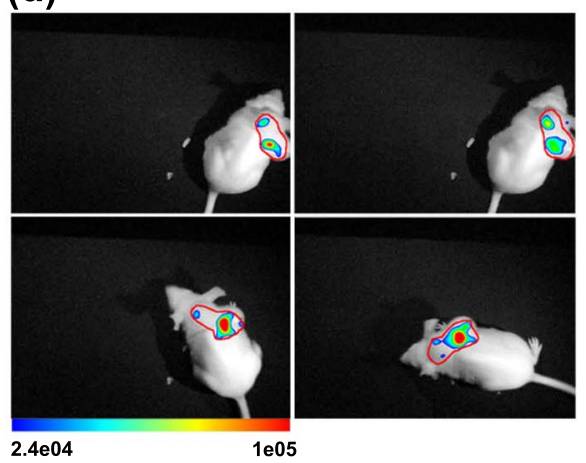

(e)

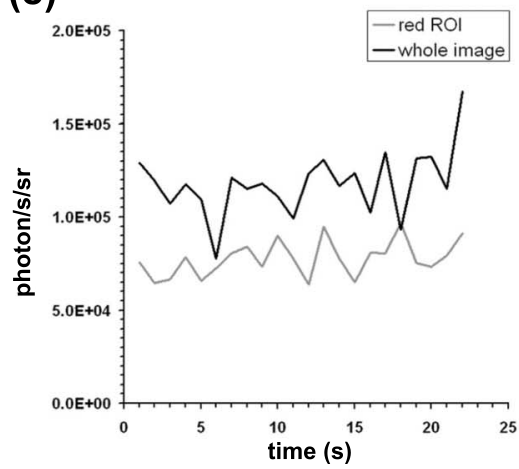

Fig. 4 Freely moving nude mouse bearing luciferase expressing tumors. (a) Illustrative sequence from Video 1. Identical ROIs respectively corresponding to the beginning (blue) and the end (red) of the acquisition. (b) Signal intensity time course for three different ROls (exposure time $1 \mathrm{~s}$ ). Signal levels (plain black and plain gray) comparable to the signal on the complete FOV (dashed). The times corresponding to the four images in (a) are indicated by the numbered arrows at the bottom of the graph. (c) Mean SNRs (five sequences of the same animal) for 40-ms to 1-s exposure times. Minimum and maximum values for each exposure time are given by vertical bars. (d) Four images of the mouse with an exposure time of $160 \mathrm{~ms}$ (5-mm smoothing; photon/s/ $\mathrm{cm}^{2} / \mathrm{sr}$ ). Red ROI following bioluminescence signal. (e) Time course of the intensity (photon/s/sr) for the red ROI and the complete FOV. (Color online only.)

the dataset. The mean SNR was around 15 for an exposure time of $40 \mathrm{~ms}$ and around 88 for 1-s exposure time images. The time course of the signal intensity was also analyzed in a sequence with an exposure time of $160 \mathrm{~ms}$, using an ROI tracking the signal area [Fig. 4(d)]. As tumors could not be enclosured with automatic detection on the video image, this ROI was defined on bioluminescent data and updated every second between 0 and $24 \mathrm{~s}$. Signal intensity was calculated every second with $160 \mathrm{~ms}$ exposure time data [Fig. 4(e)]. Light intensity was rather stable (coefficient of variation 0.14 ). The total intensity was evaluated on the complete FOV (coefficient of variation of 0.15 ) and was slightly greater than the signal intensity measured in the moving ROI.

A different measurement technique was evaluated with experiments conducted on a C57/B16 mouse expressing the luc gene. Mice were shaved before the experiment to limit light absorption by fur. Beetle luciferin $(300 \mu \mathrm{L}$ at $1.5 \mathrm{mg} / \mathrm{mL}$, Promega Corporation, Madison, Wisconsin) was IP injected in the mouse without anesthesia. Ten minutes after IP injection, the mouse was placed into the chamber and allowed to move freely. Images were acquired during several minutes (Video 2). An algorithm of contour detection (Freeman algorithm) was applied to the tracking video to define a ROI enclosing the body of the animal [Fig. 5(a)]. This postprocessing was replicated on 50 images extracted at different times of the sequence. The time course of the signal intensity corresponding to this automated ROI was then plotted [Fig. 5(b)]. The signal was as stable as the total signal measured on the whole image (coefficients of variation 0.16 and 0.14 , respectively),

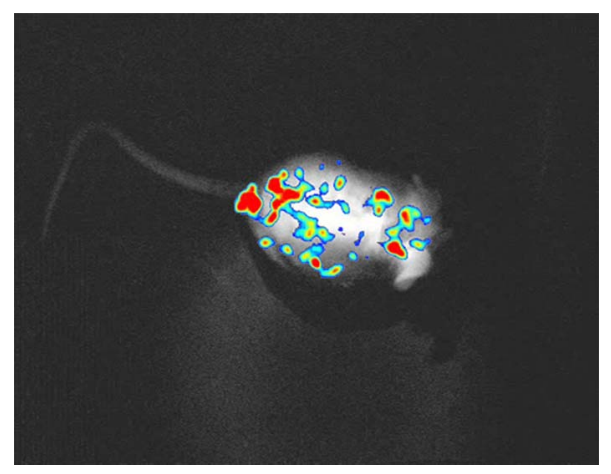

Video 2 Similar experiments to those given on Video 1 were realized with a transgenic mouse expressing luciferase. Video 2 was generated after the experiment with an exposure of $40 \mathrm{~ms}$ (CCD frame duration) and 5-mm smoothing (QuickTime, 1.94 MB).

[URL: http://dx.doi.org/10.1117/1.2976426.2]. 
(a)

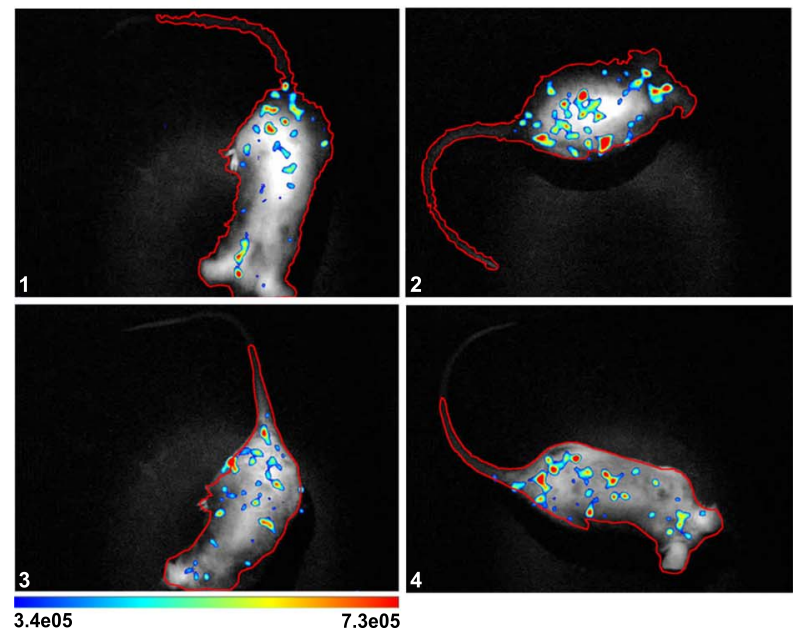

(b)

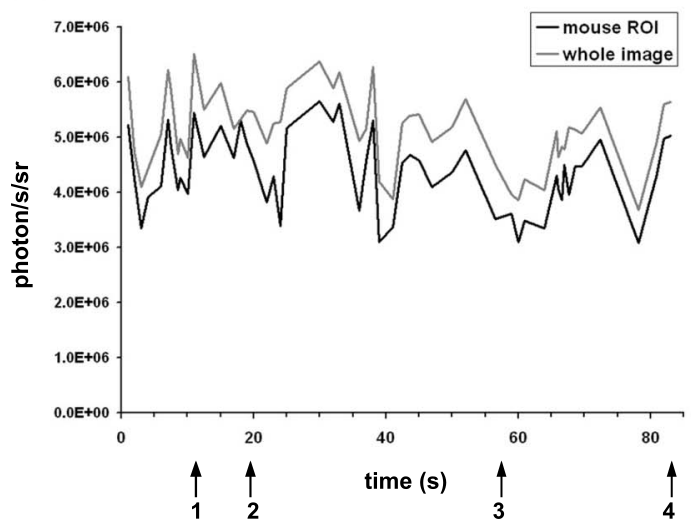

Fig. 5 Automated signal tracking on freely moving transgenic mouse. (a) Images of C57/BI6 mouse expressing luciferase extracted from Video 2. Automatic ROI in red, generated by postprocessing on video data and then used to analyze the bioluminescence on raw data. (b) Comparison of the ROI intensity time course (red ROI) and the complete FOV intensity time course for 50 images (exposure time $40 \mathrm{~ms}$ ). (Color online only.)

and intensities were comparable, showing the accuracy of the automated ROI and demonstrating that no loss of signal is generated by motion.

\subsection{Tracking Video Tests}

Finally, to analyze the influence of the exposure on the accuracy of signal measurements, the quality of the tracking was studied. Figure 6 compares the surface of an automatically generated ROI for exposure times of $5 \mathrm{~s}, 1 \mathrm{~s}$, and $160 \mathrm{~ms}$ [Fig. 6(a)]. The ROI was determined using postprocessing of bioluminescent data. Images were filtered and then segmented for automatic threshold detection of the bioluminescence signal. The resulting contour defined the ROI surface. The time course of the ROI surface for 5-s, 1-s, and 160-ms exposure time images were compared [Fig. 6(b)]. The coefficient of variation for exposure times between $5 \mathrm{~s}$ and $160 \mathrm{~ms}$ were 46,30 , and $17 \%$, respectively. It shows that a fast movement of the mouse has a strong impact on the ROI surface for 5-s and 1-s exposure time images, whereas the ROI surface mea- (a)

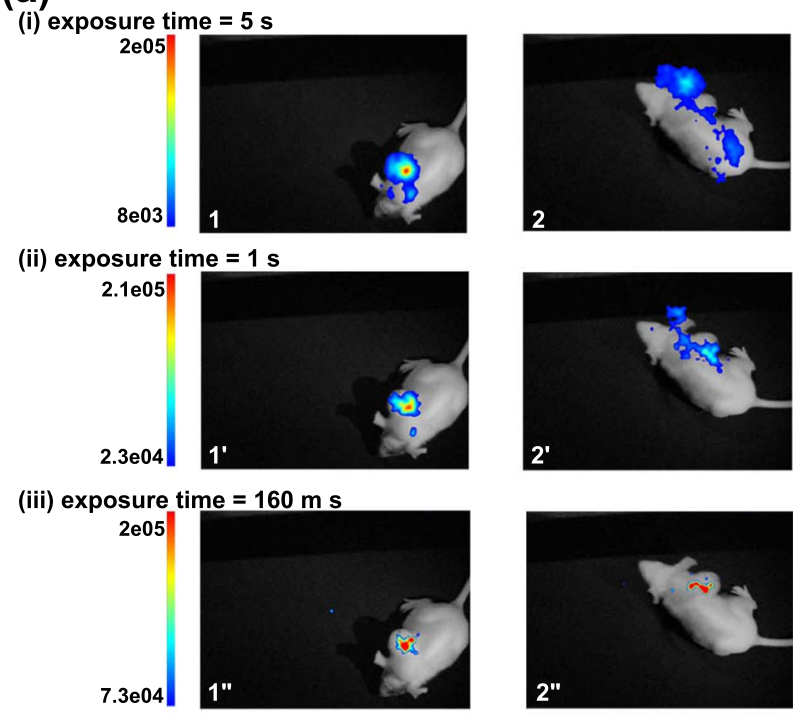

(b)

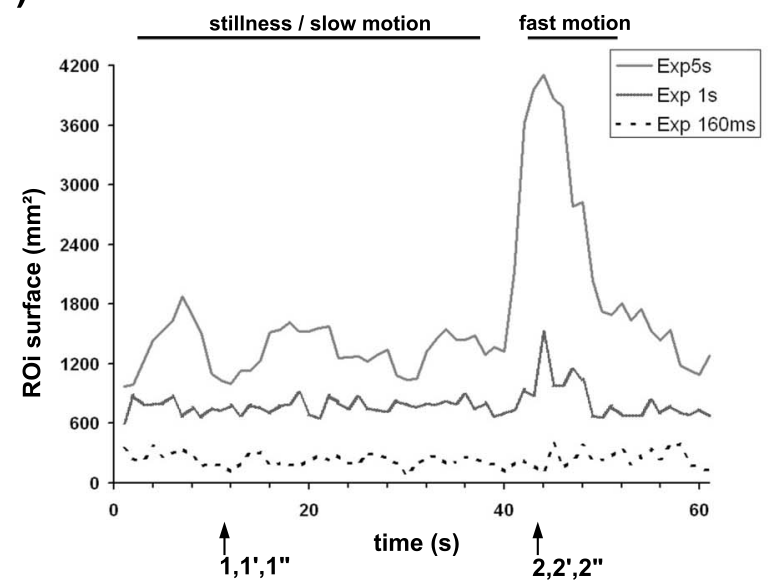

Fig. 6 Exposure time influence on registration. (a) Images of a nude mouse (5-mm smoothing; photon $/ \mathrm{s} / \mathrm{cm}^{2} / \mathrm{sr}$ ): 1 . exposure time $5 \mathrm{~s}, 2$. exposure $1 \mathrm{~s}$, and 3 . exposure time $160 \mathrm{~ms}$. Each temporal window defining the exposure time was centered on the video range of the animal. Stillness/slow motion period: $1,1^{\prime}$, and $1^{\prime \prime}$ and fast motion period: 2, 2', and 2". (b) ROI surface determined using postprocessing on bioluminescent data versus time (plain gray, plain black, and dashed) for exposure times of $5 \mathrm{~s}, 1 \mathrm{~s}$, and $160 \mathrm{~ms}$, respectively.

sured on 160-ms images is rather constant. This last temporal resolution leads to a proper location of the signal on the tumor, whereas higher exposure times lengthen the emission area along the movement direction.

\subsection{In Vivo Imaging of Endogenous Calcium Signaling in Mice}

Rogers et al. recently reported in vivo detection of $\mathrm{Ca}^{2+}$ concentration $\left(\left[\mathrm{Ca}^{2+}\right]\right)$ rises in transgenic mice expressing mitochondria-targeted GFP-aequorin. ${ }^{17}$ GFP-aequorin undergoes bioluminescence resonance energy transfer (BRET) in response to rapid $\left[\mathrm{Ca}^{2+}\right]$ variations and emits visible light with a maximum at $508 \mathrm{~nm} .{ }^{18}$ Using our optical setup in concomitant BLI and video mode, rapid $\left[\mathrm{Ca}^{2+}\right]$ rises were registered noninvasively during muscle contraction in mice. ${ }^{17}$ 
(a)

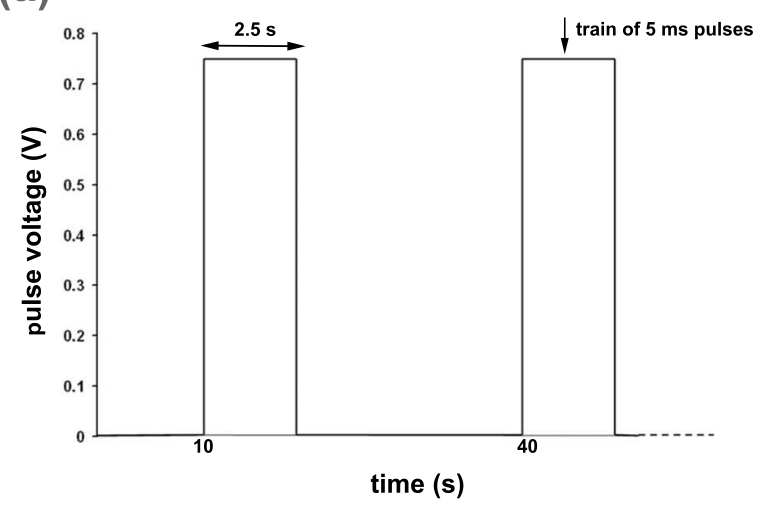

(c)

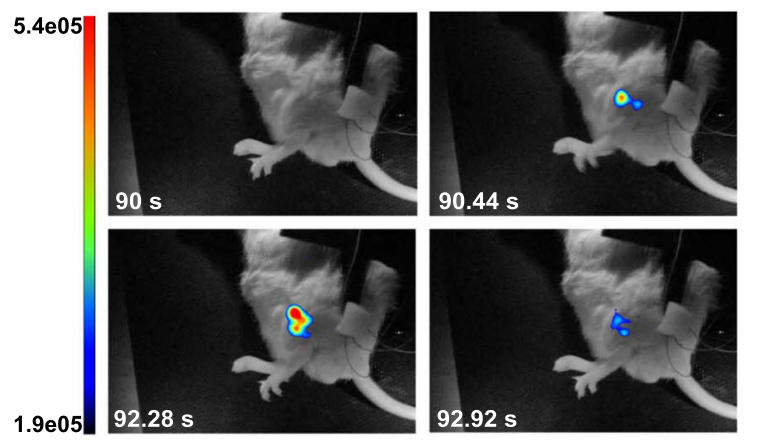

(b)

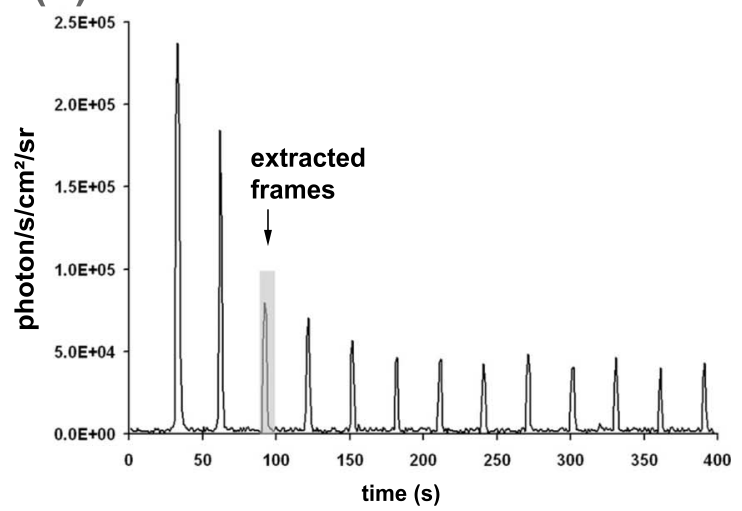

(d)

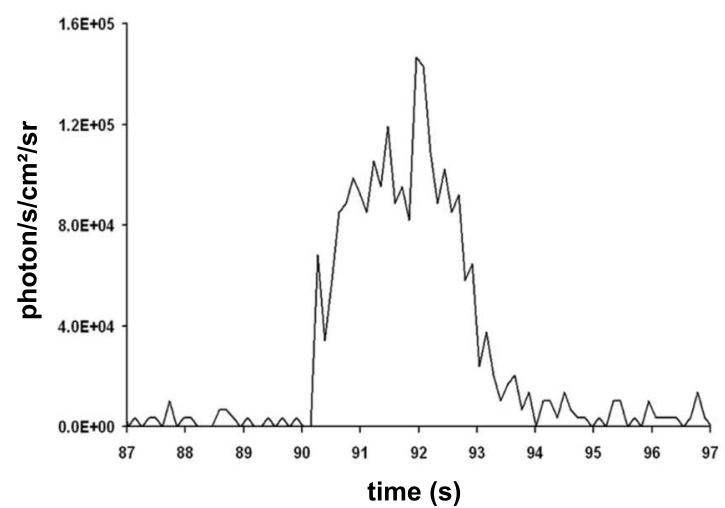

Fig. 7 In vivo detection of calcium signaling during tetanic contraction. (a) Schematic time course of the electrical stimulation. 2.5-s trains composed of 5 -ms pulses at $100 \mathrm{~Hz}$ were applied every $30 \mathrm{~s}$. The voltage of $0.75 \mathrm{~V}$ was chosen empirically. (b) Time course of the light radiance $\left(\mathrm{ph} / \mathrm{s} / \mathrm{cm}^{2} / \mathrm{sr}\right)$ for a ROI enclosing the hindlimb muscle (exposure time $1 \mathrm{~s}$ ). (c) Images extracted from Video 3 [time period indicated in gray on (a)]. (d) Expansion of the peak corresponding to the extracted time period (120-ms exposure time for each measurement).

Trains of stimuli $(2.5$-s duration; $100 \mathrm{~Hz}$; 5 -ms pulses; $0.75 \mathrm{~V})$ were applied every $30 \mathrm{~s}$ to the sciatic nerve to generate a contraction of the hindlimb muscle [Fig. 7(a)]. Bioluminescent emission and leg motion were simultaneously recorded in real time. Figure 7(b) represents the time course of the light radiance measured on an ROI enclosing the hindlimb muscle. Measurements were done on 120-ms exposure time frames. As expected from previous work, trains of stimuli generated peaks of light emission with highly reproducible kinetics. Images in Fig. 7(c) are extracted from Video 3 [corresponding time period indicated in Fig. 7(b)] and illustrate the peak of light during a tetanic contraction occurring when a train of stimuli is applied to the sciatic nerve. Figure $7(\mathrm{~d})$ shows an expansion of the third peak of graph Fig. 7(b). Faster $\mathrm{Ca}^{2+}$ transients generated by single twitch muscle contractions at lower frequencies $(1$ to $5 \mathrm{~Hz})$ could also be recorded.

\section{Discussion}

Anaesthesia can interfere with signalization pathways and motion is required in experiments such as behavioral studies and investigation of neuromuscular diseases. A system for whole body in vivo imaging of unrestrained or moving animals could limit the use of anaesthetics and enable such stud- ies. We therefore developed an imaging device that allows: 1 . bioluminescence signals to be recorded by a photon-counting CCD camera, and 2. the coregistration of this signal with a video image from a second CCD camera under infrared lighting.

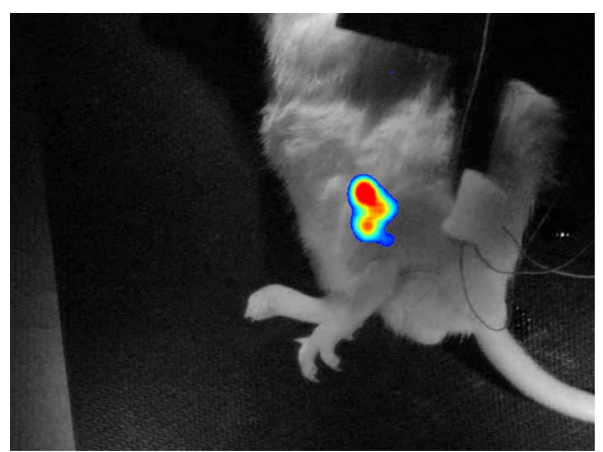

Video 3 Calcium signaling observed in vivo using a mtGa mouse. An electrode was attached to the sciatic nerve to stimulate the hindlimb muscle. Stimuli generated a peak of light correlated to muscle contraction. Exposure time is $120 \mathrm{~ms}$ and $5-\mathrm{mm}$ smoothing has been applied to BLI data (QuickTime, 1.54 MB).

[URL: http://dx.doi.org/10.1117/1.2976426.3]. 
Conversely to cooled CCDs, the photon counting technique used here enables fast kinetic imaging, which makes this method the most convenient for imaging moving animals.

To generate a noncontact tracking of the animal, the same intensified CCD camera could have been used sequentially for the bioluminescent recording and the tracking of the animal using photographic images under pulsed lighting, provided that the switching between these two modes is fast enough to be compatible with movement. This type of acquisition would have avoided a decrease of signal-to-noise ratio during the bioluminescence recording time period. Nevertheless, it would also have generated a loss of signal during the photographic image period.

In contrary, the use of continuous lighting and a second camera to record the video image enables us to record bioluminescent signals on the complete duration of the acquisition. The technical issue of the implementation of such a system in BLI modality is the overlap between the spectral sensitivity of the bioluminescence sensor in the visible range and the lighting wavelength. Thermographic or ultraviolet (UV) sensitive cameras associated with adapted illuminations could have been used to avoid spectral overlap. However, thermographic cameras are very expensive and UV light would generate autofluorescence from the skin of the animal. ${ }^{19,20}$

Our system uses a continuous near-infrared (NIR) illumination and a NIR sensitive CCD camera for animal tracking, while the bioluminescence signal is recorded continuously by the ICCD. The spectral overlap between the NIR illumination and the ICCD spectral sensitivity is compensated by filtering the illumination in front of the ICCD, and has the advantage of avoiding additional markers for tracking the animal. We use an infrared cut-off filter based on the interferential technology that has been designed to provide a blocking of six optical densities over the range [700 to $850 \mathrm{~nm}$ ]. The multilayer structure of interferential filters and their high sensitivity to angle of incidence ${ }^{21}$ makes it difficult to maintain strong blocking on a broad range. Therefore, the residual noise and the loss of transmission lead to a decrease of SNR of $30 \%$.

Results show that in spite of the SNR decrease, our system provides robust video monitoring without exogenous markers, recorded along with the signal of interest without disruption at a rate of 25 images/s.

Not surprisingly, motion induces limitations, and three main quantification issues can be stated. First, the usual measurement protocols for luminescent data analysis are difficult to apply to moving light emission areas. This issue is illustrated by intensity variation and signal loss observed when no automatic detection of the outline of the body could be realized with the video image.

Second, significant displacements of the animal during movement generate a blurring of the luminescent data. A long exposure time associated with a high amplitude movement prevents frames to be correctly registered with the video image. The accuracy of the source location on the body is therefore intrinsically related to the 2-D displacements contained in the plane defined by the stage. Mouse speed was evaluated on different motion patterns (body stretching, leaps) and the average speed was about $7 \mathrm{~cm} / \mathrm{s}$. A source point will therefore undergo a delay of $2.8 \mathrm{~mm}$ for the minimal exposure time of $40 \mathrm{~ms}$ when the signal is integrated over a window centered on an image at any particular moment. This blurring is compatible with spatial resolution of luminescent-based imaging techniques, but due to the low levels of light encountered in bioluminescence emission, exposure time may be increased to reveal the signal. This deteriorates the accuracy of signal localization with respect to the animal body. To facilitate signal measurements in cases of high exposure time, postprocessing treatments could improve the localization of the signal at the surface of the animal body and automatic ROI design could be implemented.

Third, given that the mouse movements can have a vertical component, there is a quantification defect related to the modification of the orientation of the light source with respect to the camera. This orientation issue is inherent to planar imaging, and implies an alteration of the photometry that can explain variations in measured radiances. 3-D monitoring could bring information about the vertical movement of the animal and be useful for the correction of quantification defects due to this motion.

\section{Conclusion}

We demonstrate the development of a device dedicated to whole body imaging of moving animals at high time resolution. This system is based on photon counting and includes a video monitoring function to track the animal.

This new instrument allows BLI imaging where anesthetics are suspected to cause physiological interference, such as studies monitoring tumor growth or calcium signaling. Even under anesthesia, it can be useful to monitor the position of a mouse and check its physiological state without interrupting the acquisition, a critical point if kinetic measurements are in progress. Sensitivity is sufficient to enable the detection of low intensity signals in the whole body of small animals, and temporal resolution $(40 \mathrm{~ms})$ allows the registration of physiological answers to stimuli. Finally, using the same setup, the sensitivity and temporal resolution could be improved by using a faster and more sensitive camera.

\section{Acknowledgments}

The authors would like to acknowledge the contributions of Raphael Boisgard, Frédéric Ducongé, Karine Gombert, Carine Pestourie, and Thomas Viel (CEA-LIME and INSERM U803, Paris, France) for in vitro and in vivo experiments, and Philippe Brulet (Institut Pasteur, Paris, France) for providing the GA mice. Roncali and Savinaud have a scholarship from Biospace Lab. This work was supported by the EMIL and DIMI European Networks to Tavitian.

\section{References}

1. M. Herbin, R. Hackert, J. P. Gasc, and S. Renous, "Gait parameters of treadmill versus overground locomotion in mouse," Behav. Brain Res. 181, 173-179 (2007).

2. H. Wei, B. Kang, W. Wei, G. Liang, Q. C. Meng, Y. Li, and R. G Eckenhoff, "Isoflurance and sevoflurane affect cell survival and BCL2/BAX ratio differently," Brain Res. 1037, 139-147 (2005).

3. C. Baudelet and B. Gallez, "Effect of anesthesia on the signal intensity in tumors using BOLD-MRI: Comparison with flow measurements by laser Doppler flowmetry and oxygen measurements by luminescence-based probes," Magn. Reson. Imaging 22, 905-912 (2004).

4. H. Toyama, M. Ichise, J. S. Liow, D. C. Vines, N. M. Seneca, K. J. Modell, J. Seidelb, M. V. Green, and R. B. Innis, "Evaluation of 
anesthesia effects on $[18 \mathrm{~F}] \mathrm{FDG}$ uptake in mouse brain and heart using small animal PET," Nucl. Med. Biol. 31, 251-256 (2004).

5. R. N. Sachdev, G. C. Champney, H. Lee, R. R. Price, D. R. Pickens, V. L. Morgan, J. D. Stefansic, P. Melzer, and F. F. Ebner, "Experimental model for functional magnetic resonance imaging of somatic sensory cortex in the unanesthetized rat," Neuroimage 19(3), 742750 (2006).

6. P. D. Skoubis, V. Hradil, C. L. Chin, Y. Luo, G. B. Fox, and S. McGaraughty, "Mapping brain activity following administration of a nicotinic acetylcholine receptor agonist, ABT-594, using functional magnetic resonance imaging in awake rats," Neuroscience (Oxford) 137(2), 583-591 (2006).

7. A. G. Weisenberger, B. Kross, S. Majewski, V. Popov, M. F. Smith, V. H. Tran, J. Baba, J. Goddard, M. Pomper, and B. Tsui, "Instrumentation development of a SPECT-CT system to image awake mice," Nucl. Sci. Symp. Conf. Rec., vol. 5, pp. 3000-3003 (2006).

8. F. Helmchen, M. S. Fee, D. W. Tank, and W. Denk, "A miniature head-mounted two-photon microscope: high-resolution brain imaging in freely moving animals," Neuron 31, 903-912 (2001).

9. R. Weissleder, "Scaling down imaging: molecular mapping of cancer in mice," Nat. Rev. Cancer 2(1), 1-8 (2002).

10. J. R. De Wet, K. V. Wood, D. R. Helinski, and M. DeLuca, "Cloning of firefly luciferase cDNA and the expression of active luciferase in escherichia coli," Proc. Natl. Acad. Sci. U.S.A. 82(23), 7870-7873 (1985).

11. S. Inouye and F. I. Tsuji, "Aequorea green fluorescent protein: expression of the gene and fluorescence characteristics of the recombinant protein," Fed. Eur. Biochem. Soc. Lett. 341(2-3), 277-280 (1994).

12. S. Bhaumik and S. S. Gambhir, "Optical imaging of Renilla luciferase reporter gene expression in living mice," Proc. Natl. Acad. Sci. U.S.A. 99(1), 377-382 (2001).
13. O. Shimomura, "The discovery of aequorin and green fluorescent protein," J. Microsc. 217(1), 3-15 (2005).

14. C. Leclerc, M. Lee, S. E. Webb, M. Moreau, and A. L. Millerb, "Calcium transients triggered by planar signals induce the expression of ZIC3 gene during neural induction in Xenopus," Dev. Biol. 261, 381-390 (2003).

15. L. J. van Vliet, F. R. Boddeke, D. Sudar, and I. T. Young, "Image detectors for digital image microscopy," Chap. 2 in Digital Image Analysis of Microbes; Imaging, Morphometry, Fluorometry and Motility Techniques and Applications, Modern Microbiological Methods, M. H. F. Wilkinson and F. Schut, Eds., John Wiley and Sons, Chichester, UK (1998).

16. J. L. Gach, O. Hernandez, J. Boulesteix, P. Amram, O. Boissin, C. Carignan, O. Garrido, M. Marcelin, G. O. Stlin, H. Plana, and R. Rampazzo, "Fabry-Pérot observations using a new GaAs photon counting system," Publ. Astron. Soc. Pac. 114, 1043-1050 (2002).

17. K. L. Rogers, S. Picaud, E. Roncali, R. Boisgard, C. Colasante, J. Stinnakre, B. Tavitian, and P. Brûlet, "Non-invasive in vivo imaging of calcium signaling in mice," PLoS ONE 2(10), e974 (2007).

18. V. Baubet, H. Le Mouellic, A. K. Campbell, E. Lucas-Meunier, P. Fossier, and P. Brûlet, "Chimeric green fluorescent protein-aequorin as bioluminescent $\mathrm{Ca}^{2+}$ reprters at the single-cell level," Proc. Natl. Acad. Sci. U.S.A. 97(13), 7260-7265 (2000).

19. M. Monici, "Cell and tissue autofluorescence research and diagnostic applications," Biotechnol. Annu. Rev. 11(1), 227-256 (2005).

20. J. Sandby-Moller, E. Thieden, P. A. Philipsen, J. Heydenreich, and H. C. Wulf, "Skin autofluorescence as a biological UVR dosimeter," Photodermatol. Photoimmunol. Photomed. 1(1), 33-40 (2004).

21. J. M. Saurel and J. Roig, "Properties of Perot-Fabry interferential filters illuminated in oblique incidence," J. Opt. 10(4), 179-193 (1979). 